\title{
High-Pressure Injection Injury of the Finger is not a Trivial Trauma
}

\author{
Lucas Jozef Steverlynck ${ }^{1}$, Jan Frans Noyez ${ }^{2}$ \\ Departments of ${ }^{1}$ Emergency and ${ }^{2}$ Orthopaedics, AZ Delta, Roeselare, Belgium.
}

\section{Corresponding Author:}

Dr Lucas Jozef Steverlynck

Email: lucas.steverlynck@azdelta.be

This is an Open Access article distributed under the terms of the Creative Commons Attribution License (creativecommons.org/ licenses/by/3.0).

Received

Accepted

Published

December 1, 2020

March 26, 2021

May 15, 2021

\begin{abstract}
Background: The extent of damage of a high-pressure injury is often underestimated. Most commonly, only a minor skin lesion is seen on clinical presentation. Frequent complications include amputation, septicemia and long working incapacity. On this topic, only recommendations made on bundled case reports are existent. Case Report: We describe a high-pressure latex injection injury of the finger. On clinical examination a small entry point lesion in the finger was seen. Surgical debridement showed extensive wound infiltration of latex paint. The patient could restart his work activities after 6 weeks. Conclusion: The low threshold for exploratory surgery should be maintained in high-pressure injuries.
\end{abstract}

Keywords: Amputation, Debridement, Injections, Latex, Paint, Sepsis.

\section{Introduction}

A high-pressure injection injury is an insidious lesion. The incidence of amputation ranges between $30-50 \%$ without adequate treatment. These kinds of injuries are relatively uncommon. They occur in 1 of 600 cases of all hand injuries. Minor entry point lesions frequently stand in striking contrast to extended tissue damage beneath the skin since several mechanisms cause irreversible tissue damage. In the first place, the injection force needs to be taken into account. This can cause traumatic dissection along the neurovascular bundles, compression, vasospasm, tissue ischemia, thrombosis and compartment syndrome. Another significant aspect is the chemical destruction caused by the injected fluid. It can cause cytolysis and tissue destruction, necrosis and extensive inflammatory responses [1,2]. Most tissue damage is caused by organic solvents such as (car) paint, white spirit, gasoline and oil [3]. In addition, the volume of the injected product and infection also plays an important role [1-3]. The aim of this article, is to investigate the devastating consequences of high-pressure injection injury accompanied by details on debridement and to emphasize the need to have a low threshold for surgery.

\section{Case Report}

A 40-year-old man presented to the emergency department several hours after he sustained a highpressure injury to his left middle finger, inflicted by a latex paint-gun. Pain was progressively increasing. There was no relevant past medical history. A punctiform injection lesion was noticed on the volar site of the distal interphalangeal joint [Fig.1]. There was some slight reddishness and swelling, causing reduced range of motion. Neurovascular examination was normal. The extent of the injury was assessed by ultrasound evaluation of the finger, showing a hyperechogenic irregular zone surrounded by some edema [Fig.2]. Per-operative X-rays showed radiolucent marking of the injected substance. Surgical debridement was advised by the hand surgeon. Surgery was performed under general anesthesia utilizing a garrot and a Bruner incision. Extensive wound infiltration of the white latex paint around P2 and P3 was visible [Fig.3]. A minimal flexor tendon 


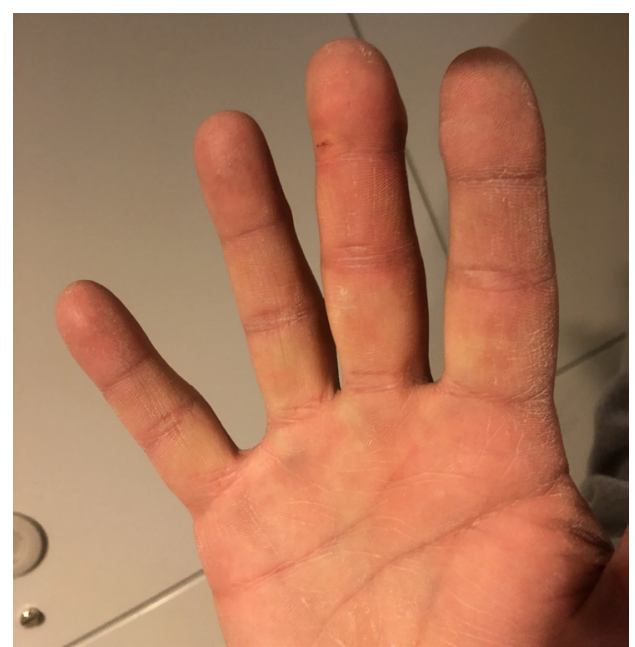

Fig.1: Aspect of the middle finger on presentation in the emergency department. Note the minimal punctiform injection site between $P 2$ and P3.

tenolysis without harming the pulleys and minimal debridement took place in order to exclude latex paint infiltration in the flexor tendon sheet. Since the exploration could rule out latex infiltration at this level, irrigation of the tendon sheet was judged not necessary. The non-surgical treatment consisted of: paracetamol and ibuprofen, triaxis vaccination for tetanus prophylaxis, elevation of the wound and post-operative amoxi-clavulanic acid during 7 days. Post-operatively, antibiotic treatment, intensive physiotherapy and revalidation followed. After 6 weeks, the patient could resume his profession but complaints of residual finger morning stiffness remained.

\section{Discussion}

The key decision in high-pressure injection injury, is the need for surgical intervention. Imaging can be useful to help assessing the size of the lesion in the presence of air or radiolucent material [4]. These injuries tend to swell. It is essential to evaluate the necessity for exploration before applying dressings under tension or stitching the wound. It is extremely important not to use ice and to avoid ring anesthesia during debridement as this promotes vasospasm and vascular compression [5]. All injected and necrotic material must be
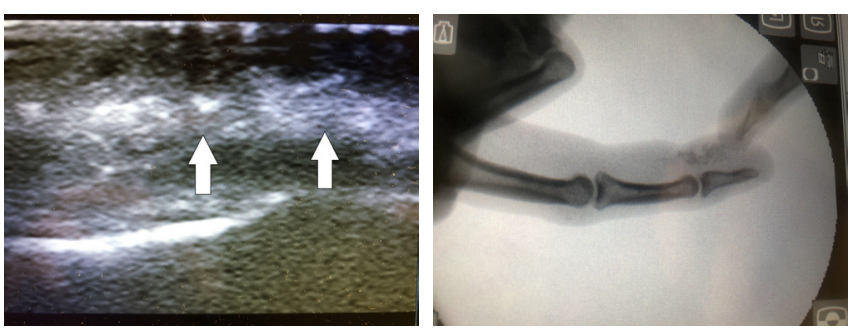

Fig.2: Quick look ultrasound in the emergency department shows a hyperechogenic irregular zone (arrows) (2A). Peroperative $X$-ray of the middle finger (2B).

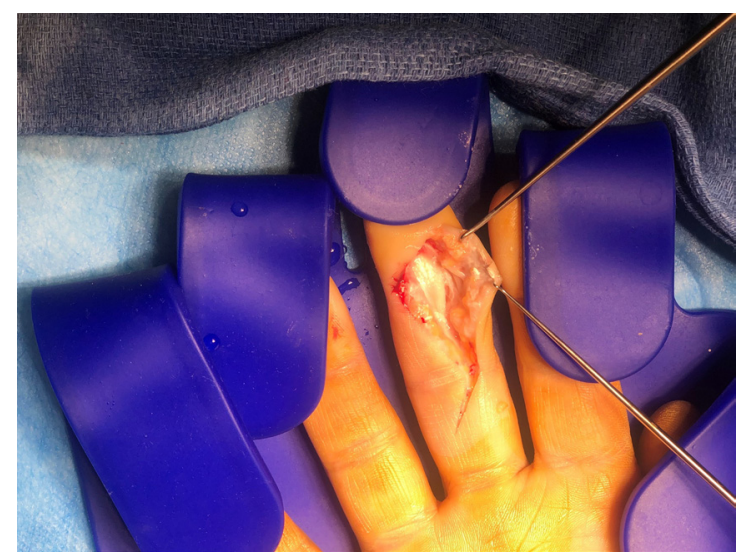

Fig.3: Per-operative findings after Bruner incision, showed extensive wound infiltration of white latex between P2 and P3.

removed. When applying a tourniquet, don't wrap the arm with an Esmarch bandage in order to avoid further spreading of the injected material $[1,2]$. Wide exposure of the injected area needs to be taken into account through extensile exposures (either mid-lateral or Bruner).

Debridement of all dead and devitalized tissue is indicated while preserving longitudinal neurovascular structures of the digit as well as A2 and A4 pulleys if possible. Extensive saline irrigation of all exposed tissues is of primary importance, working only by gravity, avoiding pulse lavage irrigation $[5,6]$. Solvents should not be used during debridement as they have cytolytic properties $[1,2]$. Evaluate the need to release the carpal canal and/or Guyon canal if indicated. The last two cornerstones in therapy are prophylactic antibiotic administration and control of the tetanus 
status [5]. There is no evidence that corticoids have an impact on prevention of infectious complications and on the avoidance of amputation $[1,2]$.

In the post-operative phase, repetitive debridement should be considered as well as durable soft tissue cover using flaps or grafts [5]. There is no consensus on optimal timing of performing the initial debridement. However, early surgical exploration is recommended, especially if faced with cytotoxic material or in case of extensive soft tissue damage [5]. Paints and solvents are more irritating substances and have larger cytolytic properties than water, some oils and greases [1,2]. Exposure to organic solvents effects a higher risk of amputation [4]. Latex paints based on water have less destructive properties [1]. The location of the injection injury also influences the risk of amputation. Fingers have a six-fold greater risk of amputation compared to palm and thumb. Injections into the thumb and little finger are more likely to involve the forearm because of their connection with the radial and ulnar bursae. Injection of the index, middle, and ring fingers usually remains confined to the tendon sheath where there is limited space [5].

Rehabilitation takes a long time. Active and active-assisted physiotherapy should be started as early as possible. Exercising active and passive range of motion stressing the tendon gliding is extremely important [5]. A resumption of work is, on average, possible 7.5 months after the accident. Only $43 \%$ of the patients can resume their previous job. $17 \%$ of all patients are continuously unable to work [4]. In these circumstances prevention plays a key role. The physical and economic consequences are severe, especially in the absence of an immediate and adequate therapy. On average, the interval between a high-pressure injection injury and first medical consult is nine hours. This reflects the need for more awareness for these lesions.

The importance of preventive measures is crucial: safety education and informing users of high-pressure guns, point at the severity of these injuries and last but not least, appropriate protective clothing [1].

\section{Conclusion}

High-pressure injection injuries are rare but not trivial. Initial clinical examination is often misleading, especially if only a minor skin lesion can be seen. If not treated appropriate, complications may lead to amputation. To avoid this, a low threshold for early surgical treatment should be adapted.

Contributors: LJS: manuscript writing, patient management; JFN: manuscript editing, patient management; LJS will act as a study guarantor. Both authors approved the final version of this manuscript and are responsible for all aspects of this study.

Funding: None; Competing interests: None stated.

\section{References}

1. Verhoeven N, Hierner R. High-pressure injection injuries of the hand: an often-underestimated trauma: case report with study of the literature. Strategies Trauma Limb Reconstr. 2008;3:27-33.

2. Rosenwasser MP, Wei DH. High-pressure injection injuries to the hand. J Am Acad Orthop Surg. 2014;22:3845.

3. Pappou IP, Deal DN. High-pressure injection injuries. J Hand Surg Am. 2012;37: 2404-2407.

4. Amsdell SL, Hammert WC. High-pressure injection injuries in the hand: current treatment concepts. Plast Reconstr Surg Glob Open. 2013;132(4):586e-91e.

5. Hadeed A, Anhony JH, Hoffler CE. Hand high pressure injury. NCBI Bookshelf. 2020; July. Available at: https:// www.ncbi.nlm.nih.gov/books/NBK542210/. Accessed November 24, 2020. 